\title{
Kinetics of the Hydrolytic Cleavage of Organic Protolytes
}

\author{
BERTIL HA NSEN
}

\author{
Research Institute of National Defence, Dept. 1, Sundbyberg 4, Sweden
}

\begin{abstract}
A method for the investigation of the hydrolytic cleavage of organic protolytes is described. It is based on automatic titrations at constant $\mathrm{pH}$. The mathematical treatment is given. By using a "curve fitting" method the reaction rate constants of the basic and acidic forms of the protolyte and its dissociation constant can be calculated. The method is tested with the hydrobromide of 2-dimethylaminoethyl-thiolopropionate.
\end{abstract}

$\mathrm{T}_{\mathrm{r}}$

The most common method for measuring the alkaline hydrolysis of a compound is to dissolve the compound in excess alkali and then after different long times titrate the alkali not used up in the reaction. In another wellknown method the reaction takes place in a buffer solution with the $\mathrm{pH}$ remaining almost constant. The reaction can be followed in various ways, e.g. by taking optical measurements of the concentration of one of the reaction products as a function of time.

When the compound to be hydrolyzed is a protolyte as, for instance, an ester of an amino acid or a mono ester of a di-basic acid, the composition of the reaction mixture becomes rather complicated. Because of protolysis the compound may exist simultaneously in both acidic and basic forms which react with different rates. For the determination of the corresponding rate constants it is important to know the dissociation constant of the compound. But if the reactions not are so slow that they can be neglected in the buffer zone of the compound, it will be difficult or even impossible to measure the dissociation constant by common methods. This means that we may have three constants which must be regarded as unknowns in all calculations. If the first of the two experimental methods mentioned above is used, the mathematical treatment will be very laborious. When choosing the buffer method the theoretical treatment becomes rather simple, if it is valid to assume that the ions of the solution do not affect the reactions. Unfortunately this is seldom true..$^{1,2}$

This paper describes how the hydrolysis of a protolyte can be investigated according to a method which does not seem to have been used before. It is based on an automatic titration of the reaction mixture so that the $\mathrm{pH}$ is 
maintained constant.$^{3}$ During the reaction the consumption of titrant is recorded, and the curve so obtained is used for the calculation of the reaction velocity. The reaction is carried out at several $\mathrm{pH}$-values, and the resulting rate constants are plotted in a diagram. By comparing this (Fig. 1) with a family of standard curves (Fig. 2) it is possible to find the velocity constants of the hydrolysis of the two forms of the protolyte as well as its dissociation constant. The method is very similar to the buffer method but has the advantage of no complicated effects due to the ions of the solution.

\section{THEORY}

A mathematical expression of the alkaline hydrolysis of a protolyte is deduced on the following assumptions.

1) The hydrogen ion activity is constant during the whole reaction.

2) All experiments are carried out at the same temperature.

3) For each of the acidic and basic forms of the compound the reactions with molecules and ions other than the hydroxyl ion can be neglected. That means that there are only two reactions running in parallel, and both are of the second order.

4) The ionic strength is the same in all experiments, and during the reaction it changes only slightly. For that reason the activity factors according to the Debye-Hückel theory can be regarded as constants.

The deduction here is carried out for an ester containing an amino group in its alcohol part, because an ester of this kind was used in the experiments described later in the paper. The result can, however, easily be applied to other classes of compounds.

$$
\text { S y m bols }
$$

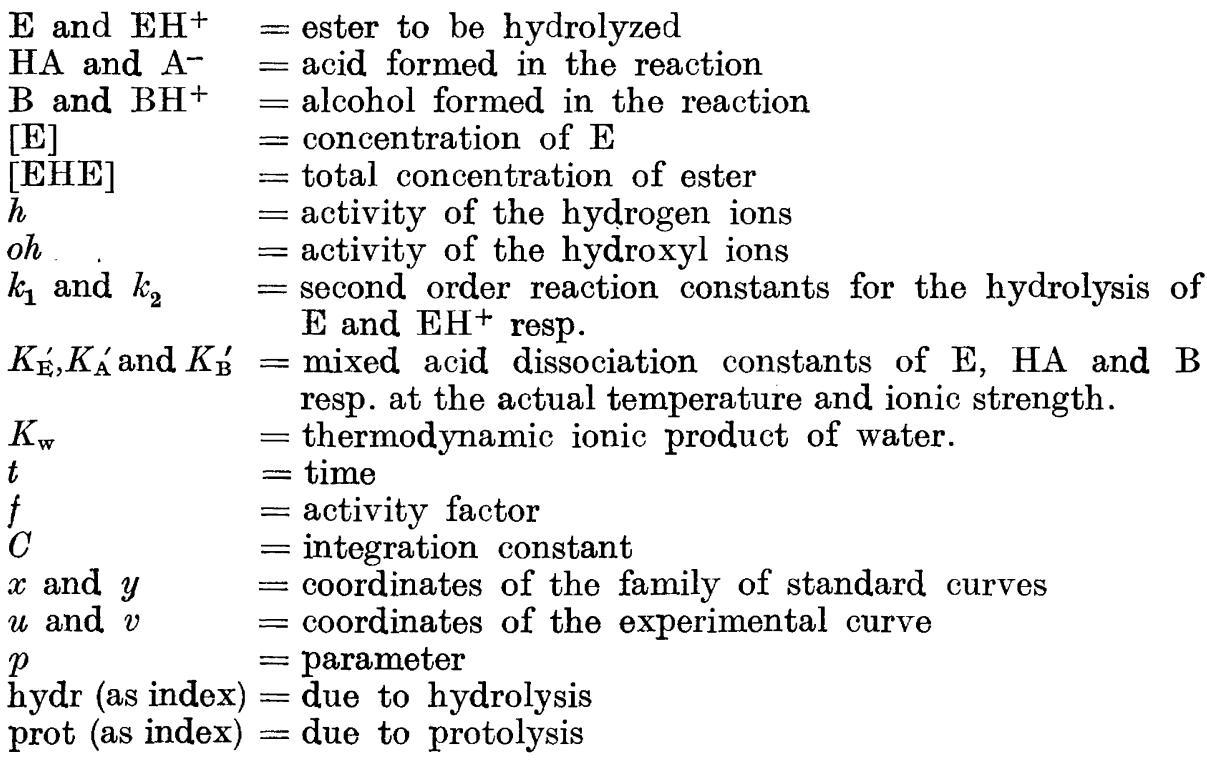


The protolytic equilibria of the solution are

$$
\begin{array}{lll}
\mathrm{E}+\mathrm{H}^{+} \rightleftharpoons \mathrm{EH}^{+} & \text {(1) } & \mathrm{A}^{-}+\mathrm{H}^{+} \rightleftharpoons \mathrm{HA} \\
\mathrm{B}+\mathrm{H}^{+} \rightleftharpoons \mathrm{BH}^{+} & \text {(3) } & \mathrm{H}^{+}+\mathrm{OH}^{-} \rightleftharpoons \mathrm{H}_{2} \mathrm{O}
\end{array}
$$

They are determined by the equations

$$
\begin{array}{lll}
\frac{[\mathrm{E}] \cdot h}{\left[\mathrm{EH}^{+}\right]}=K_{\mathrm{E}}^{\prime} & (5) & \frac{\left[\mathrm{A}^{-}\right] \cdot h}{[\mathrm{HA}]}=K_{\mathrm{A}}^{\prime} \\
\frac{[\mathrm{B}] \cdot h}{\left[\mathrm{BH}^{+}\right]}=K_{\mathrm{B}}^{\prime} & \text { (7) } & h \cdot o h=K_{\mathrm{w}}
\end{array}
$$

The reactions between the hydroxyl ions and $\mathrm{E}$ and $\mathrm{EH}^{+}$may be written

$$
\begin{aligned}
& \mathrm{E}+\mathrm{OH}^{-} \stackrel{k_{1}}{\longrightarrow} \mathrm{A}^{-}+\mathrm{B} \\
& \mathrm{EH}^{+}+\mathrm{OH}^{-} \stackrel{k_{2}}{\longrightarrow} \mathrm{A}^{-}+\mathrm{BH}^{+}
\end{aligned}
$$

These reactions must be followed by immediate changes in the equilibria (1)(4).

When differentiating the expression for the total concentration of the ester

$$
[\mathrm{EHE}]=[\mathrm{E}]+\left[\mathrm{EH}^{+}\right]
$$

we get

$$
d[\mathrm{EHE}]=d[\mathrm{E}]+d\left[\mathrm{EH}^{+}\right]
$$

The changes of $[\mathrm{E}]$ and $\left[\mathrm{EH}^{+}\right]$are due partly to the reactions (9) and (10) and partly to the changes in the equilibrium (1). We may then write

$$
\begin{aligned}
& d[\mathrm{E}]=d[\mathrm{E}]_{\text {hydr }}+\mathrm{d}[\mathrm{E}]_{\text {prot }} \\
& \mathrm{d}\left[\mathrm{EH}^{+}\right]=\mathrm{d}\left[\mathrm{EH}^{+}\right]_{\text {hydr }}+\mathrm{d}\left[\mathrm{EH}^{+}\right]_{\text {prot }}
\end{aligned}
$$

The protolytic change (1) gives

$$
\mathrm{d}[\mathrm{E}]_{\mathrm{prot}}=-\mathrm{d}\left[\mathrm{EH}^{+}\right]_{\mathrm{prot}}
$$

If we now add up (12)-(15) we see that

$$
\mathrm{d}[\mathrm{EHE}]=\mathrm{d}[\mathrm{E}]_{\mathrm{hydr}}+\mathrm{d}\left[\mathrm{EH}^{+}\right]_{\mathrm{hydr}}
$$

The hydrolysis reactions (9) and (10) give us the following differential equations

$$
\begin{aligned}
& -\mathrm{d}[\mathrm{E}]_{\mathrm{hydr}}=k_{1} \cdot[\mathrm{E}] \cdot\left[\mathrm{OH}^{-}\right] \cdot \mathrm{d} t \\
& -\mathrm{d}\left[\mathrm{EH}^{+}\right]_{\mathrm{hydr}}=k_{\mathbf{2}} \cdot\left[\mathrm{EH}^{+}\right] \cdot\left[\mathrm{OH}^{-}\right] \cdot \mathrm{d} t
\end{aligned}
$$

According to assumption 1 (p. 325)

and eqn. (5) written

$$
h=\text { constant }
$$

$$
[\mathrm{E}] \cdot \frac{h}{K_{\mathrm{E}}^{\prime}}=\left[\mathrm{EH}^{+}\right]
$$

then shows that $[\mathrm{E}]$ is directly proportional to $\left[\mathrm{EH}^{+}\right]$independently of (9) and $(10)$. 
Using (20) when combining (16)-(18) we get

According to (11) and (20)

$$
-\mathrm{d}[\mathrm{EHE}]=\left(k_{1}+k_{2} \cdot \frac{h}{K_{\mathrm{E}}^{\prime}}\right) \cdot[\mathrm{E}] \cdot\left[\mathrm{OH}^{-}\right] \cdot \mathrm{d} t
$$

and consequently

$$
[\mathrm{E}]=\frac{[\mathrm{EHE}]}{1+\frac{h}{K_{\mathrm{E}}^{\prime}}}
$$

$-\mathrm{d}[\mathrm{EHE}]=\frac{k_{1}+k_{2} \cdot \frac{h}{K_{\mathrm{E}}^{\prime}}}{1+\frac{h}{K_{\mathrm{E}}^{\prime}}}[\mathrm{EHE}] \cdot\left[\mathrm{OH}^{-}\right] \cdot \mathrm{d} t=k_{3} \cdot[\mathrm{EHE}] \cdot\left[\mathrm{OH}^{-}\right] \cdot \mathrm{d} t$

where

$$
k_{3}=\frac{k_{1}+k_{2} \cdot \frac{h}{K_{\mathrm{E}}^{\prime}}}{1+\frac{h}{K_{\mathrm{E}}^{\prime}}}
$$

Because of assumption 4 (p. 325) and the eqns. (8) and (19) we have

$$
\left[\mathrm{OH}^{-}\right]=\frac{o h}{f_{\mathrm{OH}^{-}}}=\text {constant }
$$

The only variables of (23) are consequently [EHE] and $t$. The integration of (23) gives

$$
\ln [\mathrm{EHE}]=-k_{3} \cdot\left[\mathrm{OH}^{-}\right] \cdot t+C
$$

We see that the hydrolysis can be treated as a reaction of the first order, and the rate constant $k_{3} \cdot\left[\mathrm{OH}^{-}\right]$contains the three unknowns $k_{1}, k_{2}$ and $K_{\mathrm{E}}^{\prime}$.

If $k_{3}$ is determined at three different $h$-values and the obtained sets of values, $\left(h_{\mathrm{a}}, k_{3 \mathrm{a}}\right),\left(h_{\mathrm{b}}, k_{3 \mathrm{~b}}\right)$ and $\left(h_{\mathrm{c}}, k_{3 \mathrm{c}}\right)$, are inserted into $(24)$, we get an equation system which has solutions with respect to $k_{1}, k_{2}$ and $K_{\mathrm{E}}^{\prime}$. However, the expressions obtained have no practical interest because they consist mainly of a large number of differences, which, as a rule, are small and so give rise to large errors in the calculated constants, e.g.

$$
k_{1}=\frac{h_{\mathrm{a}} h_{\mathrm{b}} k_{3 \mathrm{c}}\left(k_{3 \mathrm{a}}-k_{3 \mathrm{~b}}\right)+h_{\mathrm{b}} h_{\mathrm{c}} k_{3 \mathrm{a}}\left(k_{3 \mathrm{~b}}-k_{3 \mathrm{c}}\right)+h_{\mathrm{c}} h_{\mathrm{a}} k_{3 \mathrm{~b}}\left(k_{3 \mathrm{c}}-k_{3 \mathrm{a}}\right)}{h_{\mathrm{a}} h_{\mathrm{b}}\left(k_{3 \mathrm{a}}-k_{3 \mathrm{~b}}\right)+h_{\mathrm{b}} h_{\mathrm{c}}\left(k_{3 \mathrm{~b}}-k_{3 \mathrm{c}}\right)+h_{\mathrm{c}} h_{\mathrm{a}}\left(k_{3 \mathrm{c}}-k_{3 \mathrm{a}}\right)}
$$

In his paper on graphical methods Sillén ${ }^{4}$ emphasizes the usefulness of "curve fitting methods" for determination of, e.g., equilibrium constants, when the direct calculations give unsatisfactory accuracy. Such a method has for that reason been developed for the determination of $k_{1}, k_{2}$ and $K_{\mathrm{E}}^{\prime}$ from the sets of values $\left(h, k_{3} \cdot\left[\mathrm{OH}^{-}\right]\right)$obtained from the experiments.

Let us start by looking for a suitable family of standard curves with which an experimental curve can be compared. We shall find that a useful family is obtained from the equation

Acta Chem. Scand. 12 (1958) No. 2 


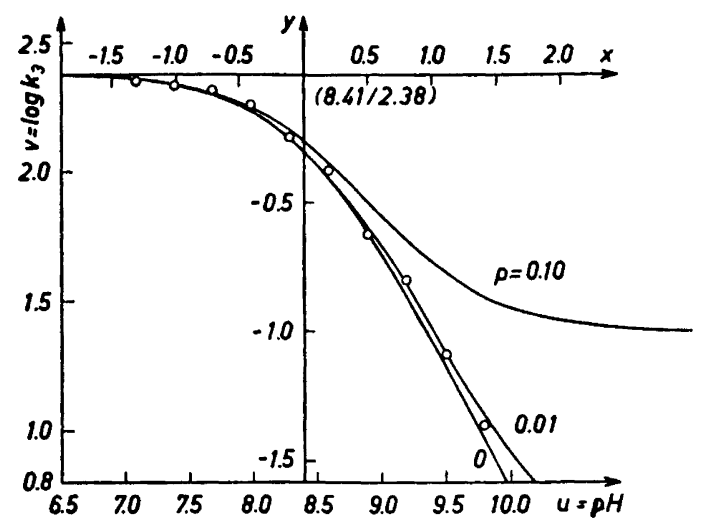

Fig. 1. The experimental values plotted in the family of curves for $u_{0}=8.41, v_{0}=2.38$.

$$
y=\log \frac{p+10^{-x}}{1+10^{-x}}
$$

by plotting the function in an (x,y)-diagram for certain values of $p$ (Fig. 2). After substituting

into (24) we get

$$
\left\{\begin{array}{l}
u=\mathrm{pH}=-\log h \\
v=\log k_{3}
\end{array}\right.
$$

$$
v=\log k_{2}+\log \frac{\frac{k_{1}}{k_{2}}+\frac{10^{-u}}{K_{\mathrm{E}}^{\prime}}}{1+\frac{10^{-u}}{K_{\mathrm{E}}^{\prime}}}
$$

The family of $(u, v)$-curves that can be obtained by giving different values to $k_{1} / k_{2}$ in (30) is the same as the family from (28) except that the origins do not coincide.

Let us now use the experimental values of $h$ and $k_{3}$ for plotting an $(u, v)$ diagram (Fig. 1). When comparing the family (28) and the experimental curve (Fig. 1), we see that, for parallel abscissae, the best fit gives a value $p=p_{0}$. As the shapes of the curves are dependent only on $p_{0}$ and $k_{1} / k_{2}$, respectively, these values must be equal

$$
p_{0}=\frac{k_{1}}{k_{2}}
$$

The curves are brought to cover each other, and the coordinates $\left(u_{0}, v_{0}\right)$ of the origin of the $(x, y)$-system are read off.

As $x=0$ must correspond to

in $(30)$, and $y=0$ to

$$
\frac{10^{-u_{0}}}{K_{\mathrm{E}}^{\prime}}=1
$$

$$
v_{0}=\log k_{2}
$$




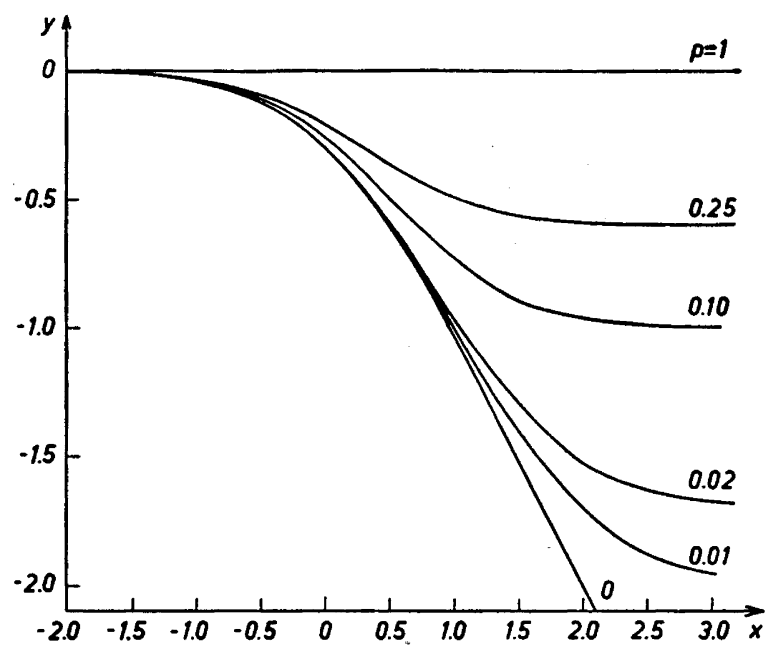

Fig. 2. Some standard curves of the family.

we have obtained three equations (31)-(33) from which $k_{1}, k_{2}$ and $K_{\mathrm{E}}^{\prime}$ can be calculated.

It is worth mentioning that the family of standard curves can be restricted to values of $p$ in the interval $0 \leqq p \leqq 1$. For $p>1$ we make the following rearrangement of (28)

which gives

$$
\left\{\begin{array}{l}
x=-x^{\prime} \\
y=y^{\prime}-\log p^{\prime} \\
p=1 / p^{\prime}
\end{array}\right.
$$

$$
y^{\prime}=\log \frac{p^{\prime}+10^{-x^{\prime}}}{1+10^{-x^{\prime}}}
$$

We see that (28) and (35) are the same equation, and as $p^{\prime}=1 / p^{\prime}$ the family of curves made up for $0 \leq p \leq 1$ also can be used for $p>1$.

This division of the family into two classes requires that, before comparing the curves, we have to find out to which of the classes the experimental curve belongs.

1. If $v$ decreases with increasing $u$ it means that $0 \leqq p \leqq 1$ and we can compare the curves directly and read off the values $p_{0}, u_{0}$ and $v_{0}$.

2. If $v$ increases with increasing $u$ we have the case $p>1$ and must first make the rearrangement of (29)

$$
\left\{\begin{array}{l}
u=-u^{\prime} \\
v=v^{\prime}
\end{array}\right.
$$

i.e. we reverse the paper on which the experimental curve is drawn. It is then possible to compare the curves and read off the values ${p_{0}^{\prime}}_{0}, u_{0}^{\prime}$ and $v_{0}^{\prime}$. Remembering that $\left(u_{0}^{\prime}, v_{0}^{\prime}\right)$ does not correspond to $x=0, y=0$ but to $x^{\prime}=0$, $y^{\prime}=0$, we can calculate $k_{1}, k_{2}$ and $K_{\mathrm{E}}^{\prime}$ from eqns. (31)-(34) and (36).

Acta Chem. Scand. 12 (1958) No. 2 


\section{EXPERIMENTAL}

The hydrolysis experiments have been carried out with the hydrobromide of 2-dimethylaminoethyl-thiolopropionate $e^{5,8}$ by using the automatic recording titrator described by Larsson and Hansen. ${ }^{3}$ The reactions took place in a nitrogen atmosphere at $25^{\circ} \pm 0.1^{\circ}$. From the recorded consumptions of $0.05 \mathrm{M}$ sodium hydroxide the first order rate constants $k_{3} \cdot\left[\mathrm{OH}^{-}\right]$were calculated according to Guggenheim.?

Assumption 4 (p. 325) was considered fulfilled by performing the reaction in $0.1 \mathrm{M}$ potassium chloride and starting with $0.6 \mathrm{mM}$ concentration of the ester.

The glass electrode used was of the type Radiometer G $202 \mathrm{~B}$ and the reference electrode a saturated calomel electrode, Radiometer K 300. The electrodes were standardized against $0.05 \mathrm{M}$ potassium hydrogen $o$-phthalate, $\mathrm{pH} 4.01$. The $\mathrm{pH}$-fluctuations during a single experiment were within $\pm 0.01 \mathrm{pH}$-units.

\section{RESULTS}

Though many of the experiments were carried out in the $\mathrm{pH}$-range where both the 2-dimethylaminoethyl-thiolopropionate and the reaction product containing the amino group have a good buffering effect the curves plotted for the calculations of $k_{3} \cdot\left[\mathrm{OH}^{-}\right]$(eqn. (26)) were straight lines. This shows that the reaction really is first order at constant $\mathrm{pH}$.

As a rule two or more experiments were carried out at each $\mathrm{pH}$. The deviations from the mean values of $k_{3} \cdot\left[\mathrm{OH}^{-}\right]$were always less than $5 \%$, which if they would be due only to the readings of the of the $\mathrm{pH}$-scale corresponds to the acceptable error of $0.02 \mathrm{pH}$-units or $1 \mathrm{mV}$.

In Fig. 1 the result was plotted according to (29) with $\mathrm{pH}$ on the abscissa and $\log k_{3}$ on the ordinate. [ $\mathrm{OH}^{-}$, which is required for the calculations of $k_{3}$ from $k_{3} \cdot\left[\mathrm{OH}^{-}\right]$, was obtained from $\mathrm{pH}$ assuming ${ }^{8} K_{\mathrm{w}}=10^{-14.00}$ and $f_{\mathrm{OH}}=0.76$ (eqn. (25)). As the plotted curve had a negative slope it was immediately comparable with the family of curves (Fig. 2). The comparison showed (Fig. 1) that we had the best fit for a small value of $p$. The exact $p_{0}$-value could not be determined because for practical reasons it was impossible to perform any experiments at $\mathrm{pH}>10$ and so the curve could not be extended far enough to the right. However, the values $u_{0}=8.41 \pm 0.01$ and $v_{0}=$ $2.38 \pm 0.01$ could be read off. The errors given were estimated from the largest movements of the curves that could be done without getting an unacceptable fit. To the errors must also be added the systematic error 0.02 which was the accuracy of the $\mathrm{pH}$-meter. The calculations of $K_{\mathrm{E}}^{\prime}$ and $k_{2}$ (eqns. (31)(33)) gave $K_{\mathrm{E}}^{\prime}=(3.9 \pm 0.3) \times 10^{-9} \mathrm{~mole} / \mathrm{l}$ and $k_{2}=(2.4 \pm 0.2) \times 10^{2} \mathrm{l} / \mathrm{mole} \cdot$ sec. Regarding $k_{1}$ we can only say that $k_{1}<<k_{2}$ because it was impossible to find a $p_{0}$-value.

Estimating ${ }^{8} f_{\mathrm{EH}^{+}}=0.76$ we can calculate the thermodynamic dissociation constant of the ester $\mathrm{p} K_{\mathrm{E}}=8.29 \pm 0.03$. From $\mathrm{pH}$-measurements of mixtures of aqueous solutions of 2-dimethylaminoethyl-thiolopropionate and its hydrobromide Tammelin ${ }^{5}$ found $\mathrm{p} K_{\mathrm{E}}=8.28 \pm 0.03$ which agrees with the value above.

It is very interesting to compare the velocity constant of the alkaline hydrolysis of the acidic form of 2-dimethylaminoethyl-thiolopropionate (I) 
and that of the corresponding quaternary compound, propionyl-thiocholine (II), which has been investigated by Heilbronn ${ }^{9}$.

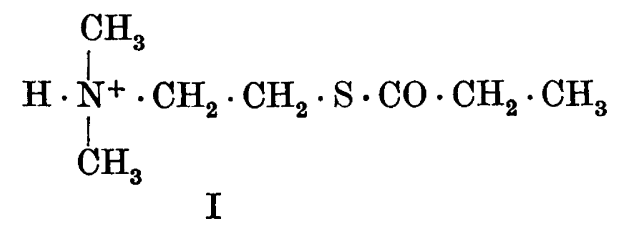<smiles>CCOC(=O)SCC[N+](C)(C)C</smiles>

Though both I and II have a positively charged ammonium group the second order rate constant of $I$ is 240 times greater than that of II. The experimental material is, however, insufficient to be used for an explanation of the difference.

To Professor G. Ljunggren, the head of this Institute, I wish to express my gratitude for his interest in this work.

\section{REFERENCES}

1. Frost, A. A. and Pearson, R. G. Kinetics and Mechanism, John Wiley \& Sons, Inc., Now York 1956, p. 138.

2. Glasstone, S., Laidler, K. J. and Eyring, H. The Theory of Rate Processes, McGrawHill Book Company Inc., New York 1941, p. 427.

3. Larsson, L. and Hansen, B. Svensk Kem. Tidskr. 68 (1956) 521.

4. Sillén, L. G. Acta Chem. Scand. 10 (1956) 186.

5. Tammelin, L.-E. Acta Chem. Scand. 11 (1957) 487.

6. Hansen, B. Acta Chem. Scand. 11 (1957) 537.

7. Guggenheim, E. A. Phil.mag. 2 (1926) 538.

8. Bates, R. G. Electrometric $p H$ determinations, John Wiley \& Sons, Inc., New York 1952 , p. 52.

9. Heilbronn, E. Personal communication.

Received November 12, 1957. 